Advocacy work focused on threats to elephants may further strengthen support for government action, but this may not significantly alter the embedded social values that fuel demand.

Finally, the study found that non-profit environmental organizations, scientists and family and friends are the most trusted sources of information on issues related to ivory. The types of organizations that are currently working to reduce demand and facilitate regulatory changes are well positioned for impact.

Although there have been other studies that have examined ivory demand, this is the first study of which we are aware that looked across five key ivory consumption countries and analysed socio-economic drivers of ivory consumption. We hope that this research can inform methods to reduce ivory demand, including policy measures, grass-roots and social marketing campaigns, consumer-focused communications and country-specific approaches. We recognize that solutions must go beyond consumer behaviour change to incorporate and address legality, corruption, law enforcement, intelligence sharing, education, capacity building and coordination, transportation and logistics, economics, livelihoods and governance. At the same time, what ultimately drives the illegal killing of elephants and the highly commercialized and criminalized trafficking of ivory are socio-economic cultural beliefs tied to the perceived value of ivory. We believe that the results of this research point to a need for transformational steps to decouple ivory and high status to make ivory purchasing socially and culturally unacceptable.

Robert Lee and Catherine Workman National Geographic Society - Science \& Exploration, Washington, DC, USA E-mail roblee@ngs.org

ERIC WHAN GlobeScan Inc., Toronto, Canada

\section{Rediscovery of the supposedly extinct Pedicularis humilis in the eastern Himalayas}

Pedicularis humilis Bonati, a perennial herb species of the family Orobanchaceae, is endemic to the Hengduan Mountains in south-west China. It was categorized as Extinct on the China Species Red List in 2013. This species had only been collected once, in 1913, by George Forrest and was described by Gustave Henri Bonati in 1921 (G.H. Bonati, 1921, Notes from the Royal Botanic Garden Edinburgh, 13, 106-107). According to the single type collection (George Forrest 11969) stored at the herbaria of the Royal Botanic Garden Edinburgh (E, holotype) and the Institute of Botany, Chinese Academy of Sciences (PE, isotype), this species is only known from the Shweli-Salween divide, in the southern Hengduan Mountains, an area of c. 400,000 ha in western Yunnan. Neither the Qinghai-Tibet Plateau
Expedition of 1982 nor the Gaoligongshan Biodiversity Survey of 1998-2007, which surveyed close to the type location and adjacent areas, located additional specimens.

With the joint support of the Chinese Union of Botanical Gardens (Grant No. KFJ-1W-NO1-12), the National Natural Science Foundation of China (Grant No. 31370243, 31570212), the Natural Science Foundation of Yunnan (Grant No. 2014FB169) and the Talent Project of Yunnan (Grant No. 2015HBo92), the Kunming Botanical Garden surveyed for any remnant $P$. humilis in the southern Hengduan Mountain range during JulyAugust in 2015. Fortunately, c. 300 individuals in flower were discovered in three separate sites in alpine meadows at an altitude of $3,200 \mathrm{~m}$. The total area of occupancy of the species is $c .100 \mathrm{~m}^{2}$. This suggests that it should be categorized as Critically Endangered on the IUCN Red List on the basis of criterion B2ab(i,ii,iii,v). Also, because of its very limited distribution, small population size and habitat degradation, it should be included in the list of PSESP (Plant Species with Extremely Small Populations) in China (Ma et al., 2013, Biodiversity and Conservation, 22, 803-809). Our survey and information obtained from interviews with local people indicated that the main threats to this species are its small population size, the high frequency of destruction by people, and road construction (and hence habitat loss). Urgent and effective measures need to be taken to protect this species.

The Kunming Botanical Garden is now carrying out further studies on the genetic diversity of $P$. humilis and its genetic relationships to other Pedicularis species of the eastern Himalayas, to obtain a better understanding of microevolution in this species. With the collaboration of the staff of nature reserves we are also planning to collect seeds of $P$. humilis for propagation and future restoration of the species in the wild. The southern Hengduan Mountains are currently managed as a nature reserve by the local government. However, the alpine meadows in this region merit additional attention because this is the only known natural habitat of the remnant $P$. humilis.

RoNG LI Key Laboratory for Plant Diversity and Biogeography of East Asia, Kunming Institute of Botany, Chinese Academy of Sciences, Kunming, China

XIAOCHUN SHI Gaoligongshan National Nature Reserve, Baoshan, China

WENBIN YU Center for Integrative Conservation, Xishuangbanna Tropical Botanical Garden, Chinese Academy of Sciences, Mengla, China

Shi Feng and Weibang Sun Kunming Botanical Garden, Kunming Institute of Botany, Chinese Academy of Sciences, Kunming, China.E-mailwbsun@mail.kib.ac.cn 